\title{
A copula-based approach for generating lattices
}

\author{
Tianyang Wang1 (D) James S. Dyer ${ }^{2}$. \\ Warren J. Hahn ${ }^{3}$
}

(C) Springer Science+Business Media New York 2015

\begin{abstract}
Discrete approximations such as binomial and trinomial lattices have been developed to model the intertemporal dynamics of variables in a way that also allows contingent decisions to be included at the appropriate increments in time. In this paper we present an approach for developing these types of models based on copulas. In addition to ease of implementation, a primary benefit of this approach is its generality, and we show that various binomial and trinomial approximation methods for valuing contingent claim securities in the literature are special cases of this approach, each based on a choice of a particular set of probability and/or branching parameters. Because this approach encompasses these and other cases as feasible solutions, we also show how it can be used to optimize the construction of lattices so that discretization error is minimized, and we demonstrate its application for an option pricing example.
\end{abstract}

Keywords Stochastic processes - Discrete models · Option pricing · Copulas

Tianyang Wang

Tianyang.Wang@business.colostate.edu

James S. Dyer

Jim.Dyer@mccombs.utexas.edu

Warren J. Hahn

Joe.Hahn@mccombs.utexas.edu

1 Department of Finance and Real Estate, Colorado State University, Fort Collins, CO 80523, USA

2 Department of Information, Risk, and Operations Management, University of Texas at Austin, Austin, TX 78712, USA

3 Department of Finance, University of Texas at Austin, Austin, TX 78712, USA 


\section{Introduction}

Many types of dynamic optimization problems are constructed over an underlying model of an uncertain state variable stochastic process, such as problems related to the valuation of financial options. Various approaches to modeling stochastic process in continuous time have been developed and implemented, such as the partial differential equation approach based on extensions of the classic work of Black and Scholes (1973). Our focus in this paper, however, is on lattice- or tree-based methods that provide a discrete approximation of the stochastic process. Lattice-based methods are well established as a tool for option valuation and other applications because of their robustness to a wide variety of contingent decisions, their ease of implementation and their intuitive appeal.

Several approaches have been developed for constructing a discrete approximation of an underlying stochastic process. The first example of this approach was a binomial lattice model that converges weakly to a geometric Brownian motion diffusion process, or GBM (Cox et al. 1979) (hereafter, CRR). This binomial model can be used to accurately approximate solutions from the Black-Scholes-Merton (hereafter, BSM) continuous-time option valuation model, but it can also be used to solve for the value of early-exercise American options, whereas the BSM model can only value European options. Various extensions to the original CRR binomial model have been proposed, including contributions by Jarrow and Rudd (1983), Tian (1993, 1999), Chen and Yang (1999), Hilliard and Schwartz (2005), and Chung and Shih (2007). The motivations for these subsequent lattice models are improving the rate of convergence or generalizing the model for more complex stochastic processes or pricing more complex derivatives.

Trinomial lattice methods have also been proposed as a generalization of binomial lattice models, and to improve approximation accuracy. Examples include models proposed by Boyle (1988), Omberg (1988), Parkinson (1977), Derman et al. (1996), Clewlow and Strickland (1998), Figlewski and Gao (1999), and Hull (2006). Some recent articles that include applications of lattice models are Baule and Wilkens (2004), Broadie and Kaya (2007), Ji and Brorsen (2011), Costabile et al. (2011), and Ji and Brorsen (2011).

While binomial lattices are generally considered to be simpler conceptually, trinomial lattices are computationally more flexible because the asset price in a trinomial tree moves in three directions compared with only two for a binomial lattice. As a result the number of time-steps in the time horizon can be reduced in a trinomial lattice to attain the same accuracy obtained by a binomial lattice (Widdicks et al. 2002). Furthermore, trinomial lattice methods can also be viewed as an explicit finite difference method, which offers considerable flexibility in the choices of grids for the time and space dimensions, and is useful for dealing with discrete dividends, barriers, and other common features (Hull 2006; Broadie and Detemple 2004; Barone-Adesi et al. 2008). Recent articles that include applications of lattice models for contingent claim asset pricing are Bizid and Jouini (2005), Chambers and Lu (2007), Jabbour et al. (2010), and Dumas and Lyasoff (2012).

The basic objective of a lattice- or tree-based method is to use discrete-time and discrete-state increments to approximate a continuous stochastic differential equation, with the accuracy of the approximation governed by the length of the time increment 
and with convergence to the continuous process guaranteed as the time increment in the discrete model approaches zero. Convergence is typically established through the use of a specification of state space increments and branching probabilities in the discrete model that is derived by matching the moments of the discrete process with the moments of the continuous process. The literature has focused on discretization of the underlying variable, such as an asset price or the log-transformed underlying variable, to construct the binomial or trinomial model.

Our framework is in the same spirit; however, we break down the approximation a step further and focus on the discretization of the Wiener process, or the standard Brownian motion component, of the underlying GBM stochastic process. We believe that this new approach to the construction of lattices provides an important synthesis of previous work that offers conceptual and practical value regarding increased transparency for this important family of computational tools. The approach leads to contributions in three areas. First, this is the first work to construct a binomial or trinomial lattice model based on copulas, a technique which allows a GBM to be represented as a series of dependent discrete probability distributions (Wang and Dyer 2012) so that lattices can be constructed in a more flexible manner than with the existing methods. Second, we show that this flexible approach is very general, and that it encompasses many of the various binomial and trinomial tree methods in the literature as special cases determined by the choices of particular sets of parameters. Third, we demonstrate how the generality of this approach allows lattice construction to be optimized, which expands the modeling possibilities beyond the discrete set of existing methods, including those mentioned above. This eliminates the need for an a-priori choice or a trial-and-error selection of a modeling method, and also supports improved computational efficiency, because tree or lattice construction can be specified under defined criteria, such as fit to the continuous distribution implied by the stochastic process.

We do acknowledge that there may be other motivations for choosing the parameters of a trinomial tree or lattice that are not included within this framework, and do not claim that every useful approximation will be a special case of our general approach. Nevertheless, this framework does provide a unifying theory that includes many of the most popular trinomial trees in the literature, that can be made into a standard algorithm which is intuitive and easy to program, and that may also enhance the abilities of students and practitioners to understand the common threads among many of the different models that have been proposed and implemented.

The rest of this paper is organized as follows: In Sect. 2, we review the two basic types of discrete approximations, both of which we will generalize in this paper. In Sect. 3, we present the general copulas-based lattice framework and discuss its relationship with various binomial and trinomial tree methods in the literature. In Sect. 4 we present numerical examples to illustrate the generality of the proposed lattice method and show how it can be optimized under two different criteria. In Sect. 5 we conclude the paper and discuss future research.

\section{Basic types of discrete approximations}

For a given probability space $(\Omega, \mathcal{F}, P)$, we consider an asset with price $S(t)$ that follows a GBM: 


$$
\frac{d S(t)}{S(t)}=(r-\delta) d t+\sigma d W(t)
$$

where $r$ is the interest rate, $\delta$ is the dividend yield, $\sigma$ is the volatility of the asset price process, and $d W(t)$ represents a Wiener process, $W(t) \sim N(0, t)$, at time $t$. Given this process, there are generally two choices for constructing a discrete approximation: (1) model $S(t)$ directly, and (2) model the $\log$ of $S(t)$.

\subsection{Discretization of GBM for $S(t)$}

The approximation developed by Boyle (1988) is an example of a discretized description of a GBM for the asset price $S(t)$. This method uses a trinomial framework, which was intended to improve upon binomial approaches, to model an underlying asset price as a recombining tree, where the price at each node has three possible paths: up, down or a stationary middle path.

The endpoint values for each path are found by multiplying the starting value in the node by the appropriate factor: $u, d$ or $m$. Given the common assumption that $m=1$, the endpoint values are $S_{u}=S u, S_{m}=S, S_{d}=S d$. The corresponding probabilities of reaching those endpoints are derived by matching the first two moments of the continuous distribution of the given GBM and requiring that they sum to one:

$$
\begin{aligned}
& p_{1}+p_{2}+p_{3}=1 \\
& p_{1} S d+p_{2} S+p_{3} S u=S e^{(r-\delta) \Delta t} \\
& p_{1}(S d)^{2}+p_{2} S^{2}+p_{3}(S u)^{2}=S^{2} e^{(r-\delta)^{2} \Delta t^{2}}\left(e^{\sigma^{2} \Delta t}-1\right) .
\end{aligned}
$$

Letting $u=e^{\lambda \sigma \sqrt{\Delta t}}, m=1$, and $d=e^{-\lambda \sigma \sqrt{\Delta t}}=\frac{1}{u}$ so that the structure is recombining, and solving the above system of three equations yields the three branching probabilities:

$$
p_{1}=\frac{1}{2 \lambda^{2}}-\frac{\nu \sqrt{\Delta t}}{2 \lambda \sigma} ; p_{2}=1-\frac{1}{\lambda^{2}} ; p_{3}=\frac{1}{2 \lambda^{2}}+\frac{\nu \sqrt{\Delta t}}{2 \lambda \sigma},
$$

where $v=r-\delta-\frac{1}{2} \sigma^{2}$ and $\lambda$ is greater than 1 . By using different values of $\lambda$, a range of values of $u$ may be obtained. The parameter $\lambda$ can be used to adjust a particular asset node to a convenient level; e.g., to coincide with a strike or barrier in an option valuation problem. As an example, Hull (2006) specifies a value $\lambda=\sqrt{3}$ so that $u=e^{\sigma \sqrt{3 \Delta t}}, m=1, d=e^{-\sigma \sqrt{3 \Delta t}}=\frac{1}{u}$ and

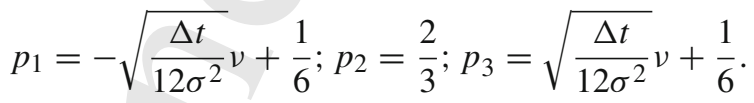

\subsection{Discretization of $\ln (S(t))$}

If $S(t)$ follows the GBM described above, Ito's lemma can be applied to obtain the process for $x(t)=\ln (S(t)): \frac{d x(t)}{x(t)}=v d t+\sigma d W(t)$. Therefore, the natural log- 
arithm of $S(t)$ is normally distributed with the following parameters: $\ln (S(t)) \sim$ $N\left(\ln (S(0))+v t, \sigma^{2} t\right)$.

As an alternative to the approximation scheme discussed above, a trinomial approximation to $n(S(t))$ can be constructed. An example of this approach is the trinomial lattice model proposed by Clewlow and Strickland (1998). In this model, over a small time increment $\Delta t$, the log of asset price can go down by $\Delta x$ (the state variable increment), stay the same, or go up by $\Delta x$, with probabilities $p_{1}, p_{2}$, and $p_{3}$ respectively.

The drift and volatility parameters of the continuous time process in this development are captured by $\Delta x, p_{1}, p_{2}$, and $p_{3}$. The increment $\Delta x$ cannot be chosen independently and was specified in this case as $\Delta x=\sigma \sqrt{3 \Delta t}$. As in the previous example, the relationship between the parameters of the continuous time process and the discrete trinomial approximation are obtained by matching the first two moments of $x$ and requiring that the probabilities sum to one. Solving these equations yields:

$$
\begin{aligned}
& p_{1}=\frac{1}{2}\left(\frac{\sigma^{2} \Delta t+v^{2} \Delta t^{2}}{\Delta x^{2}}-\frac{v \Delta t}{\Delta x}\right) ; p_{2}=1-\frac{\sigma^{2} \Delta t+v^{2} \Delta t^{2}}{\Delta x^{2}} ; \\
& p_{3}=\frac{1}{2}\left(\frac{\sigma^{2} \Delta t+v^{2} \Delta t^{2}}{\Delta x^{2}}+\frac{v \Delta t}{\Delta x}\right) .
\end{aligned}
$$

\section{A general trinomial discretization approach}

Given these two types of basic discretization approaches, we propose a general discretization scheme for modeling a GBM process. We will show that this scheme includes various binomial and trinomial tree methods in the literature as special cases with particular specifications of the parameters of this model.

Our approach to modeling the process for a variable $S(t)$ can use the form $S(t)=$ $S(0) e^{v t+\sigma W(t)}$ or the form $x(t)=\ln (S(t))=\ln (S(0))+v t+\sigma W(t)$, and starts with the Wiener process term $W(t) \sim N(0, t)$. This approach is justified by the following Theorem.

Theorem 1 A trinomial lattice constructed by moment matching of the GBM or log transformed GBM is equivalent to moment matching of the underlying Wiener process.

$a$. The moments of the asset price $S(t)$ are a function of the corresponding moments of the Wiener process:

$$
E\left(S(t)^{n}\right)=S(0)^{n} \mathrm{e}^{v t n} \times\left(M_{W(t)}(z)\right)^{\sigma^{2}},
$$

where $M_{W(t)}(z)$ is the moment generating function of $W(t)$.

$b$. The moments of the log transformed asset price $x(t)=\ln (S(t))$ are a function of the corresponding moments of the Wiener process:

$$
E\left(x(t)^{n}\right)=[\ln (S(0))+v t]^{n}+\sigma^{n} E\left((W(t))^{n}\right) .
$$

The proof of this Theorem is provided in "Appendix 1". 
This theorem suggests the following strategy for constructing a trinomial lattice. If the discrete approximation of the Wiener process is constructed so that the first $n$ moments are matched in a trinomial lattice, then a trinomial lattice can be constructed to match the corresponding first $n$ moments for the process for $S(t)$ or the log-transformed process $x(t)=\ln (S(t))$.

We now discuss the details of how to construct a trinomial lattice for a Wiener process which we represent by $\left(W_{t_{1}}, \ldots, W_{t_{n}}\right)$ where $W_{t_{i}}, i \in(1, \ldots, n)$ represents the lattice representation of the distribution of the Weiner process in period $i$. Since this process is auto-correlated, the covariance matrix for any discrete times $t_{i}$ and $t_{j}, 0<t_{i}<t_{j}<\mathrm{T}$ is $\operatorname{Cov}\left(W_{t_{i}}, W_{t_{j}}\right)=t_{i}$. As shown by Glasserman (2003), the covariance matrix $C$ of $\left(W_{t_{1}}, \ldots, W_{t_{n}}\right)$ is then defined by $C_{i, j}=\min \left(t_{i}, t_{j}\right)$. The correlation matrix $\Sigma$ of $\left(W_{t_{1}}, \ldots, W_{t_{n}}\right)$ is therefore $\Sigma_{i, j}=\frac{\min \left(t_{i}, t_{j}\right)}{\sqrt{t_{i}} \sqrt{t_{j}}}$.

The vector $\left(W_{t_{1}}, \ldots, W_{t_{n}}\right)$ has the distribution $N(0, C)$, so the Wiener process can be constructed as a multivariate normal distribution. We do this by utilizing multivariate normal copulas, $C_{N}\left(u_{1}, \ldots, u_{n}\right)=\Phi_{\Sigma}\left(\Phi^{-1}\left(u_{1}\right), \ldots, \Phi^{-1}\left(u_{n}\right)\right)$, where $u_{1}, \ldots, u_{\mathrm{n}}$ are simple uniform variables on $[0,1]$ and $\Phi$ is the Cumulative Distribution Function (CDF) of a standard normal distribution. These copulas are convenient, since they define the underlying dependency structure of the multivariate normal distribution in terms of a Pearson product moment correlation matrix $\Sigma$.

This approach can be implemented through the normal-copula based dependent tree method (Wang and Dyer 2012) which uses the unified underlying normal copula with the uniform variables as an auxiliary step for transforming arbitrary marginal distributions, and then transforms the underlying copulas into a desired decision tree model. The uniform variables associated with the normal copula based dependent tree are:

$$
u_{i=} \Phi\left(A_{i 1} \Phi^{-1}\left(\alpha_{1}\right)+\cdots+A_{i(i-1)} \Phi^{-1}\left(\alpha_{i-1}\right)+A_{i(i)} \Phi^{-1}\left(\alpha_{i}\right)\right),
$$

where $A_{i j}$ is the element of the Cholesky factorization that decomposes the covariance matrix $\Sigma$ as $\Sigma=A A^{T}$ to give the lower triangular matrix $A=\left(A_{i j}\right)_{i, j=1}^{n}$ and $\alpha_{i}$ is the percentile of the conditional distribution $X_{i} \mid X_{1}, \ldots, X_{i-1}$.

For $\Sigma_{i, j}=\frac{\min \left(t_{i}, t_{j}\right)}{\sqrt{t_{i} \sqrt{t_{j}}}}$, the Cholesky factor is given by

$$
A=\left(\begin{array}{ccc}
\frac{\sqrt{t_{1}}}{\sqrt{t_{1}}} & 0 \ldots & 0 \\
\frac{\sqrt{t_{1}}}{\sqrt{t_{2}}} & \frac{\sqrt{t_{2}-t_{1}}}{\sqrt{t_{2}}} \ldots & 0 \\
\vdots & \vdots & \vdots \\
\frac{\sqrt{t_{1}}}{\sqrt{t_{n}}} & \frac{\sqrt{t_{2}-t_{1}}}{\sqrt{t_{n}}} \ldots & \frac{\sqrt{t_{n}-t_{n-1}}}{\sqrt{t_{n}}}
\end{array}\right)
$$

Using the same time increment in the trinomial tree structure, $\sqrt{t_{1}}=\sqrt{t_{i}-t_{i-1}}=$ $\sqrt{t_{j}-t_{j-1}}=\Delta t$ for all $i, j$, the Cholesky decomposition $A$ of the Wiener process has a special structure: the decomposition factor will be a constant $\frac{\sqrt{t_{1}}}{\sqrt{t_{i}}}$ for each row. This special feature can be used in constructing the trinomial lattice of the Wiener process. 


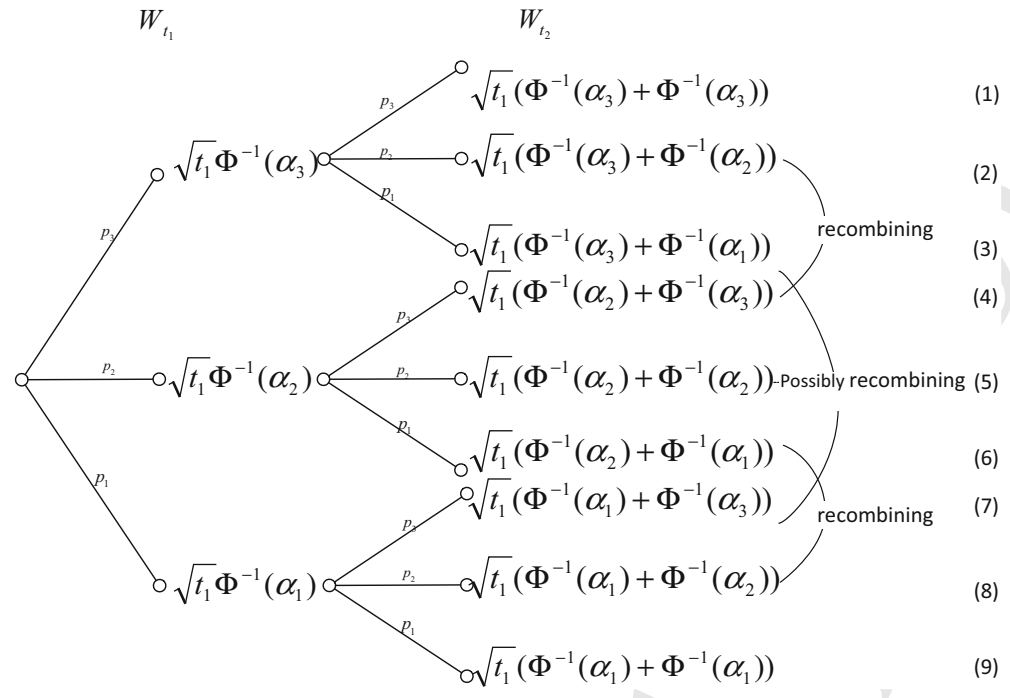

Fig. 1 Two step discretization of Wiener process

Implementing the Cholesky decomposition using the uniform variables formula gives the following expression for the underlying uniform variables:

$$
u_{i=} \Phi\left(\frac{\sqrt{t_{1}}}{\sqrt{t_{i}}} \Phi^{-1}\left(\alpha_{1}\right)+\cdots+\frac{\sqrt{t_{1}}}{\sqrt{t_{i}}} \Phi^{-1}\left(\alpha_{i-1}\right)+\frac{\sqrt{t_{1}}}{\sqrt{t_{i}}} \Phi^{-1}\left(\alpha_{i}\right)\right) \text {. }
$$

Applying the marginal transformation $\left(W_{t_{i}} \sim N\left(0, t_{i}\right)\right)$ to the underlying uniform variables $u_{i}$ results in the following representation of the Wiener process:

$$
\begin{aligned}
W_{t_{i}} & =\sqrt{t_{i}} \Phi^{-1}\left(u_{i}\right)=\sqrt{t_{i}} \frac{\sqrt{t_{1}}}{\sqrt{t_{i}}}\left(\Phi^{-1}\left(\alpha_{1}\right)+\cdots+\Phi^{-1}\left(\alpha_{i-1}\right)+\Phi^{-1}\left(\alpha_{i}\right)\right) \\
& =\sqrt{t_{1}}\left(\Phi^{-1}\left(\alpha_{1}\right)+\cdots+\Phi^{-1}\left(\alpha_{i-1}\right)+\Phi^{-1}\left(\alpha_{i}\right)\right) .
\end{aligned}
$$

This discretization is illustrated in Fig. 1.

In this structure, $p_{1}+p_{2}+p_{3}=1$, and the combination of $\alpha_{1}, \alpha_{2}, \alpha_{3}$ and $p_{1}, p_{2}$, $p_{3}$ should be selected to represent a good discretization of a normal distribution by matching the first two moments for example.

This tree structure could be naturally extended to include $W_{t_{2}}$, the Wiener process for time increment 2, as shown in Fig. 1. Because the dependent tree structure is a sequence of conditional probability distributions of $W_{t_{1}}$ and $W_{t_{2}}$, it grows in a nonrecombining manner with 3 branches of $W_{t_{2}}$ for each realization of $W_{t_{1}}$. Therefore, there are a total of 9 branches for $W_{t_{2}}$ with the endpoint values calculated as shown in Equations (1) to (9) in Fig. 1. However, because of the special structure of the Cholesky decomposition of the Wiener process, some of the branches are automatically recombining; i.e. $(2)=(4),(3)=(7)$, and $(6)=(8)$. Thus, the tree structure will become a trinomial lattice if the missing link is also connected, (3)=(5)=(7), as shown 
in the following equation $\sqrt{t_{1}}\left(\Phi^{-1}\left(\alpha_{1}\right)+\left(\Phi^{-1}\left(\alpha_{3}\right)\right)=\sqrt{t_{1}}\left(\Phi^{-1}\left(\alpha_{2}\right)+\left(\Phi^{-1}\left(\alpha_{2}\right)\right)\right.\right.$ which implies that:

$$
2 \Phi^{-1}\left(\alpha_{2}\right)=\Phi^{-1}\left(\alpha_{1}\right)+\Phi^{-1}\left(\alpha_{3}\right) .
$$

The conditional distribution of $W_{t_{2}}$ is just a normal distribution conditional on the realization of $W_{t_{1}}$. The combination of $\alpha_{1}, \alpha_{2}, \alpha_{3}$ and $p_{1}, p_{2}, p_{3}$ are selected for $W_{t_{2}}$ to provide a good discretization of a standard normal distribution, which is the same requirement for $W_{t_{1}}$, namely:

$$
\begin{aligned}
& p_{1}+p_{2}+p_{3}=1 \\
& p_{1} \Phi^{-1}\left(\alpha_{1}\right)+p_{2} \Phi^{-1}\left(\alpha_{2}\right)+p_{3} \Phi^{-1}\left(\alpha_{3}\right)=0 \\
& p_{1}\left(\Phi^{-1}\left(\alpha_{1}\right)\right)^{2}+p_{2}\left(\Phi^{-1}\left(\alpha_{2}\right)\right)^{2}+p_{3}\left(\Phi^{-1}\left(\alpha_{3}\right)\right)^{2}=1 \\
& 2 \Phi^{-1}\left(\alpha_{2}\right)=\Phi^{-1}\left(\alpha_{1}\right)+\Phi^{-1}\left(\alpha_{3}\right) .
\end{aligned}
$$

This specification includes four equations and six variables, which leaves two degrees of freedom for choosing the parameters. This also implies that there are infinitely many choices of six parameters to approximate the Wiener process using the trinomial lattice model. It is possible to make a unique choice from six parameters if restrictions are added. Many existing trinomial lattice approaches are specific cases of this general framework distinguished by the strategies for imposing restrictions on the values of these parameters.

While the approach in this paper is built on Wang and Dyer (2012) and both approaches are based on the use of copulas to capture dependence, it is important to highlight the differences between these two methods. First, the approach in Wang and Dyer (2012) is developed in a non-recombining tree structure for dependent continuous uncertainties and therefore is subject to the curse of dimensionality, as the tree size will grow exponentially with the number of uncertainties. In this paper, we develop and prove certain conditions for constructing a recombining tree/lattice which significantly extends the Wang and Dyer (2012) approach and improves its efficiency when the underlying uncertainty follows a GBM stochastic process. Second, the approach in Wang and Dyer (2012) relies on the arbitrary choice of a discretization method for each continuous uncertainty, such as extended Pearson-Tukey (EPT) method (Keefer and Bodily 1983), to choose the conditional percentile and conditional probabilities. In this work we develop the functional relationships between the conditioning percentiles and the conditional probabilities to match the moments of the underlying uncertainties. Finally, the Wang and Dyer (2012) approach was not developed to choose among alternative discretization models, whereas in this paper we demonstrate how our approach allows a lattice construction to be optimized to minimize the error in both the option price and the Greeks, which enhances the modeling possibilities.

\subsection{Symmetric Wiener lattice}

If we impose the restriction that the tree structure for the Wiener process is symmetric, since the normal distribution is symmetric, then 


$$
\Phi^{-1}\left(\alpha_{1}\right)=-\Phi^{-1}\left(\alpha_{3}\right)
$$

In that case, $2 \Phi^{-1}\left(\alpha_{2}\right)=\Phi^{-1}\left(\alpha_{1}\right)+\Phi^{-1}\left(\alpha_{3}\right)=0$, therefore

$$
\alpha_{1}=1-\alpha_{3} \text { and } \alpha_{2}=0.5 \text {. }
$$

Substituting these values into Eqs. (12) and (13) gives the following branching probabilities:

$$
p_{1}=p_{3}, \quad \text { and } \quad p_{1}=\frac{1}{2\left(\Phi^{-1}\left(\alpha_{1}\right)\right)^{2}} \text {. }
$$

Since $p_{2} \geq 0, p_{1}=p_{3}={\frac{1}{2\left(\Phi^{-1}\left(\alpha_{1}\right)\right)}}^{2} \leq 0.5$, which implies $\alpha_{1} \leq \Phi(-1)=0.1587$ to guarantee positive probabilities. Using these parameters, the constructed trinomial tree for a Weiner process is recombining, symmetric and centered at 0 , and could be written as a recombining trinomial lattice.

Now, let $W_{i, j}$ denote the value of the Wiener process in period (column) $\mathrm{i}$ and state (row) $j, i=0, \ldots, n, j=0, \ldots, 2 i+1,(j=0$ is the lowest state). Then $W_{i, 1}=i W_{1,1}$, and

$$
W_{i, j}= \begin{cases}W_{i, j-1} & j \leq 2 i \\ 0 & j=2 i+1 \\ -W_{i, 2(i+1)-j} & j>2 i+1\end{cases}
$$

As a result, the calculations in the trinomial tree or lattice are greatly simplified, because only $W_{1,1}$ the first node in period 1 of the tree, must be calculated, and the rest of the tree is simply some multiple of this value. This approach has the computational advantage of maintaining a symmetric structure for the Wiener tree through time, so the number of distinct Wiener process levels is linear in the number of time increments, $t$. This is due to the up and down moves leading back to the same level. The two period case is illustrated in Fig. 2.

Equations (16) and (17) imply that there is a unique solution for all six parameters given the specification of either $\alpha_{1}$ or $p_{1}$. This symmetric Wiener tree has the advantage that the probabilities are always constant regardless of the values of the drift and the volatility and the number of time increments.

We now show some popular discretizations from the literature that are special cases of this general framework in Table 1. The first two cases (3.1.1 and 3.1.2) are simple three-point discretizations of the normal distribution that have appeared in the literature. These two discretizations follow immediately from specifying $\alpha_{1}$ and from the use of Eqs. (16) and (17).

However, neither of these two discrete approximation methods was developed to match higher (i.e., beyond first and second) moments of the normal distribution. If the following two restrictions are added to match the third and fourth moments of the normal distribution; 
Fig. 2 Symmetric discretization as a function of $W_{1,1}$

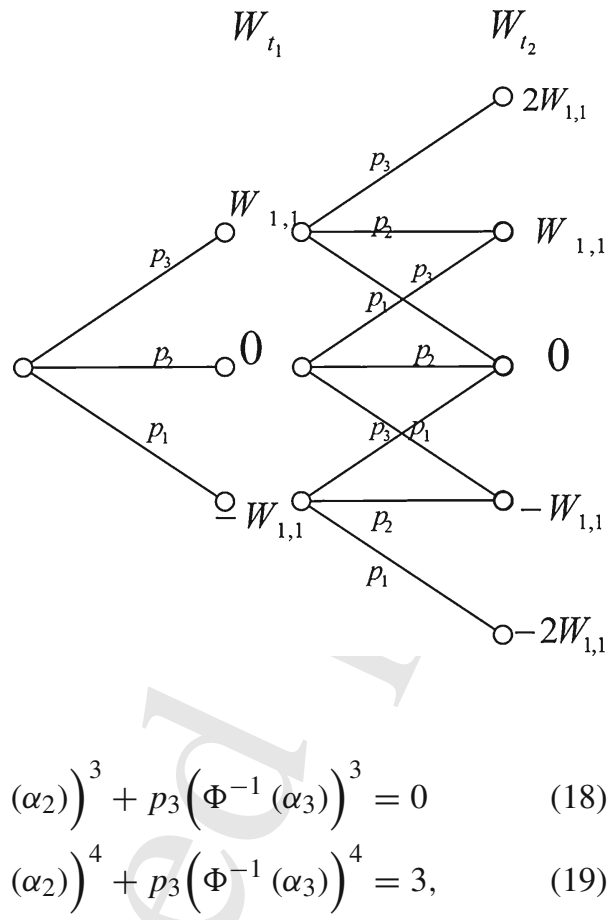

$$
\begin{aligned}
& p_{1}\left(\Phi^{-1}\left(\alpha_{1}\right)\right)^{3}+p_{2}\left(\Phi^{-1}\left(\alpha_{2}\right)\right)^{3}+p_{3}\left(\Phi^{-1}\left(\alpha_{3}\right)\right)^{3}=0 \\
& p_{1}\left(\Phi^{-1}\left(\alpha_{1}\right)\right)^{4}+p_{2}\left(\Phi^{-1}\left(\alpha_{2}\right)\right)^{4}+p_{3}\left(\Phi^{-1}\left(\alpha_{3}\right)\right)^{4}=3,
\end{aligned}
$$

then there is a unique solution set for the parameters in case 3.1.3 in Table 1: $\alpha_{1}=\Phi(-\sqrt{3}), \alpha_{2}=0.5, \alpha_{3}=\Phi(\sqrt{3}), p_{1}=p_{3}=\frac{1}{6}$, and $p_{2}=\frac{2}{3}$. These parameter values are used for the trinomial lattice methods proposed by Omberg (1988), Figlewski and Gao (1999) and Derman et al. (1996). Subsequently, Bickel et al. (2011) have shown that this approximation will match the first six moments of the normal distribution. The additional cases 3.1.4 through 3.1.8 were developed in a similar manner, as summarized in Table 1. It is important to emphasize that this ability to represent these familiar models as special cases of a general formulation is an important and unique benefit of the copula-based approach to developing lattice models.

In addition to showing that these discretization methods are a special case of our general framework, since it also matches higher moments we can illustrate how Theorem 1 applies to the trinomial lattice developed in Case 3.1.3. That is, we will show how discretizations of $W(t)$ using this special case lead to two popular discretization approaches for $\ln (S(t))$ in the literature, in accordance with the Theorem.

First, we show that the first four moments of the underlying Wiener process are matched in the Wiener lattice structure. It is easy to see that the first moment is matched because of the symmetry:

$$
p_{1} \sqrt{t} \Phi^{-1}\left(\alpha_{1}\right)+p_{2} \sqrt{t} \Phi^{-1}\left(\alpha_{2}\right)+p_{3} \sqrt{t} \Phi^{-1}\left(\alpha_{3}\right)=0
$$




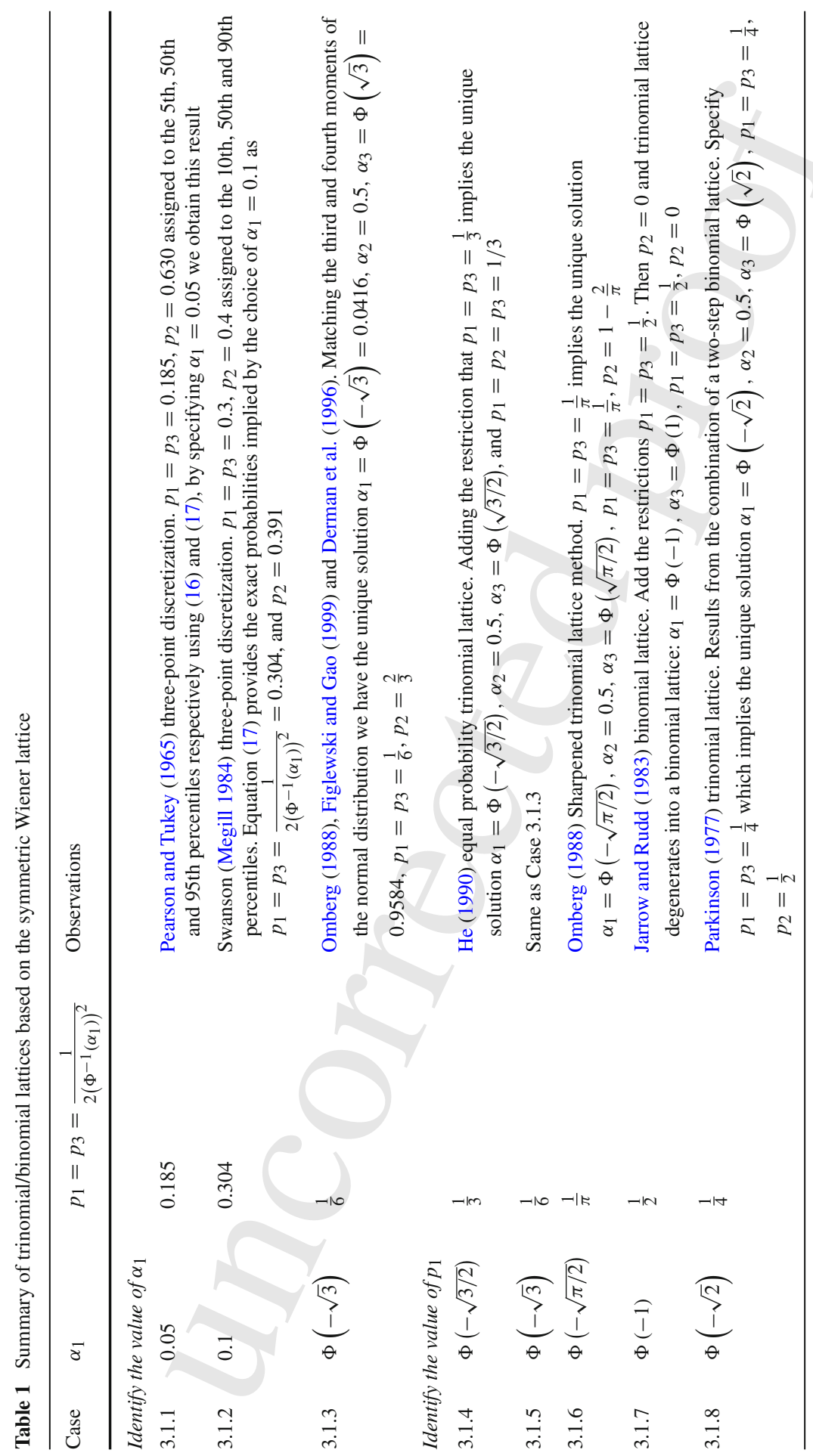


The second moment is matched by substituting the respective values for $\alpha_{i}$ and $p_{i}$ as follows:

$$
\begin{aligned}
& p_{1}\left(\sqrt{t} \Phi^{-1}\left(\alpha_{1}\right)\right)^{2}+p_{2}\left(\sqrt{t} \Phi^{-1}\left(\alpha_{2}\right)\right)^{2}+p_{3}\left(\sqrt{t} \Phi^{-1}\left(\alpha_{3}\right)\right)^{2} \\
& =p_{1} t\left(\Phi^{-1}(\Phi(-\sqrt{3}))\right)^{2}+p_{2} t\left(\Phi^{-1}(0.5)\right)^{2}+p_{3} t\left(\Phi^{-1}(\Phi(\sqrt{3}))\right)^{2} \\
& =\left(p_{1}+p_{3}\right) t\left(\Phi^{-1}(\Phi(-\sqrt{3}))\right)^{2}=\left(\frac{1}{6}+\frac{1}{6}\right) t(\sqrt{3})^{2}=t
\end{aligned}
$$

The third and forth moments are matched from the constraint Eqs. (18) and (19)

$$
\begin{aligned}
& p_{1}\left(\sqrt{t} \Phi^{-1}\left(\alpha_{1}\right)\right)^{3}+p_{2}\left(\sqrt{t} \Phi^{-1}\left(\alpha_{2}\right)\right)^{3}+p_{3}\left(\sqrt{t} \Phi^{-1}\left(\alpha_{3}\right)\right)^{3}=0 \\
& p_{1}\left(\sqrt{t} \Phi^{-1}\left(\alpha_{1}\right)\right)^{4}+p_{2}\left(\sqrt{t} \Phi^{-1}\left(\alpha_{2}\right)\right)^{4}+p_{3}\left(\sqrt{t} \Phi^{-1}\left(\alpha_{3}\right)\right)^{4}=3 t^{2} .
\end{aligned}
$$

Therefore, according to Theorem 1, the first four moments of the log transformed asset price are also matched in the lattice structure, as shown in each of the following four expressions:

$$
\begin{aligned}
E(x(t)) & =[\ln (S(0))+v t]+\sigma E((W(t)))=[\ln (S(0))+v t]+0 \\
E\left(x(t)^{2}\right) & =[\ln (S(0))+v t]^{2}+\sigma^{2} E\left((W(t))^{2}\right)=[\ln (S(0))+v t]^{2}+\sigma^{2} t \\
E\left(x(t)^{3}\right) & =[\ln (S(0))+v t]^{3}+\sigma^{n} E\left((W(t))^{3}\right)=[\ln (S(0))+v t]^{3}+0 \\
E\left(x(t)^{4}\right) & =[\ln (S(0))+v t]^{4}+\sigma^{2} E\left((W(t))^{4}\right)=[\ln (S(0))+v t]^{4}+3 \sigma^{2} t^{2} .
\end{aligned}
$$

Similarly, according to Theorem 1, we can show that the first four moments of the underlying variables are also matched. These results can be observed in both the Hull (2006) and Clewlow and Strickland (1998) trinomial lattice approaches for discretizing $\ln (S(t))$. In both cases, we show that the branching probabilities converge to the same set as above when the time increment goes to zero:

First, for Hull (2006),

$$
\lim _{\Delta t \rightarrow 0} p_{1}=\sqrt{\frac{\Delta t}{12 \sigma^{2}}} \nu+\frac{1}{6}=\frac{1}{6} ; \quad \lim _{\Delta t \rightarrow 0} p_{3}=-\sqrt{\frac{\Delta t}{12 \sigma^{2}}} \nu+\frac{1}{6}=\frac{1}{6} ; p_{2}=\frac{2}{3} .
$$

For Clewlow and Strickland (1998), the drift and volatility parameters of the continuous time process are captured by $\Delta x, p_{1}, p_{2}$, and $p_{3}$. The state space increment $\Delta x$ cannot be chosen independently, and was suggested to be $\Delta x=\sigma \sqrt{3 \Delta t}$. This choice of $\Delta x$ leads to probabilities which converge to $p_{1}=p_{3}=\frac{1}{6}$, and $p_{2}=\frac{2}{3}$ when the time increment goes to zero: 
Fig. 3 Asymmetric discretization as a function of $W_{1,1}$

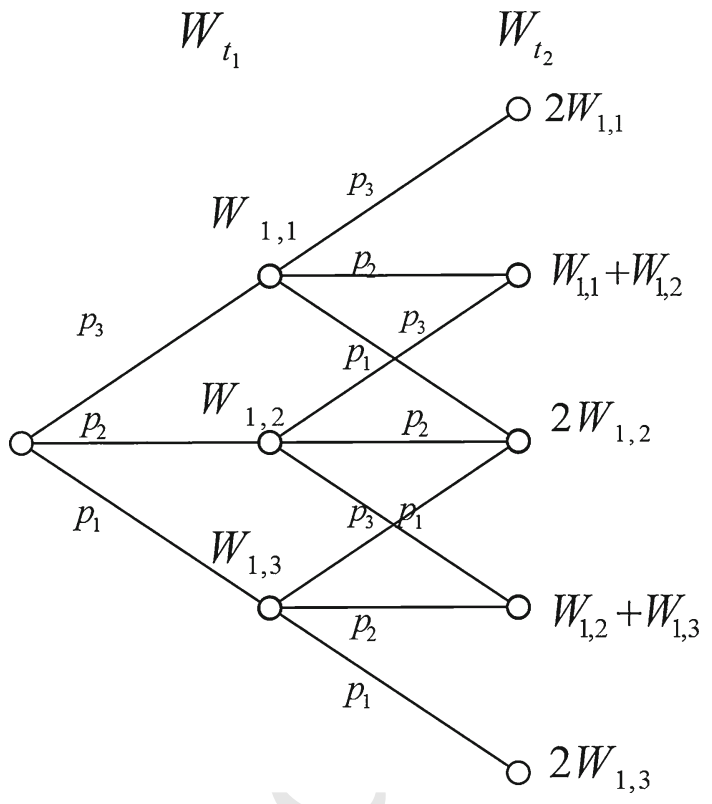

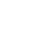$$
\lim _{\Delta t \rightarrow 0} p_{2}=\lim _{\Delta t \rightarrow 0} 1-\frac{\sigma^{2} \Delta t+v^{2} \Delta t^{2}}{\Delta x^{2}}=\frac{2}{3}
$$$$
\lim _{\Delta t \rightarrow 0} p_{3}=\lim _{\Delta t \rightarrow 0} \frac{1}{2}\left(\frac{\sigma^{2} \Delta t+v^{2} \Delta t^{2}}{\Delta x^{2}}+\frac{\nu \Delta t}{\Delta x}\right)=\frac{1}{6} .
$$

\subsection{Asymmetric Wiener lattice}

If an asymmetric Wiener tree is assumed and additional restrictions are specified to reach a unique solution, then the general framework will also include some other popular trinomial lattice approaches in the literature. The differences in the structures can be seen by comparing the endpoint values in Fig. 3 with those in Fig. 2.

Although not as simple as the case where the Wiener process approximation is symmetric, the asymmetric case still only requires the three levels of the Wiener process calculated in period one. The subsequent Wiener process levels will then be a linear function of these three levels. Again, there is a system of four equations (Eqs. 11-14) and six variables chosen to match the first two moments of the Wiener process, which leaves two degrees of freedom for choosing the parameters. This also implies that there are infinitely many choices for the six parameters to approximate the Wiener process.

Again, using Theorem 1, by constructing the discrete approximation of a Wiener process matching the first $n$ moments, an asymmetric trinomial lattice can be con- 
structed to match the corresponding first $n$ moments for the process for $S(t)$ or the log-transformed process $x(t)=\ln (S(t))$.

In some cases, it may be desirable to set a stationary middle path instead of specifying a symmetric Wiener process lattice. As before, a combination of $\alpha_{1}, \alpha_{2}, \alpha_{3}$ and $p_{1}$, $p_{2}, p_{3}$ should be selected to represent an accurate discretization of a standard normal distribution, which is the same condition as $W_{1}$. The system of matching equations in this case is:

$$
\begin{aligned}
& p_{1}+p_{2}+p_{3}=1 \\
& p_{1}\left(v \Delta t+\sigma \sqrt{\Delta t} \Phi^{-1}\left(\alpha_{1}\right)\right)+p_{2}\left(v \Delta t+\sigma \sqrt{\Delta t} \Phi^{-1}\left(\alpha_{2}\right)\right)+\cdots \\
& \quad \ldots+p_{3}\left(v \Delta t+\sigma \sqrt{\Delta t} \Phi^{-1}\left(\alpha_{3}\right)\right)=v \Delta t \\
& p_{1}\left(\left[v \Delta t+\sigma \sqrt{\Delta t} \Phi^{-1}\left(\alpha_{1}\right)\right]^{2}\right)+p_{2}\left(\left[v \Delta t+\sigma \sqrt{\Delta t} \Phi^{-1}\left(\alpha_{2}\right)\right]^{2}\right)+\cdots \\
& \quad \ldots+p_{3}\left[v \Delta t+\sigma \sqrt{\Delta t} \Phi^{-1}\left(\alpha_{3}\right)\right]^{2}=\sigma^{2} \Delta t+v^{2} \Delta t^{2} \\
& 2\left[v \Delta t+\sigma \sqrt{\Delta t} \Phi^{-1}\left(\alpha_{2}\right)\right]=\left[v \Delta t+\sigma \sqrt{\Delta t} \Phi^{-1}\left(\alpha_{1}\right)\right]+\cdots \\
& \quad \ldots+\left[v \Delta t+\sigma \sqrt{\Delta t} \Phi^{-1}\left(\alpha_{3}\right)\right] \\
& v \Delta t+\sigma \sqrt{\Delta t} \Phi^{-1}\left(\alpha_{2}\right)=0
\end{aligned}
$$

After some algebra, Eqs. (21), (22) and (23) can be reduced to the same form of Eqs. (12-14). Equation (24) specifies the stationary middle path which leads directly to the expression $\alpha_{2}=\Phi\left(-\frac{v \sqrt{\Delta t}}{\sigma}\right)$. This leaves one degree of freedom, which can be utilized in the following ways:

\section{Case 3.2.1: Matching the log-transformed moments}

If the step size $\Delta x$ for the $\log$ of $S(t)$ or the factor $u$ for $S(t)$ is chosen, then since $\Delta x=\ln u$,

$\left.\left[v \Delta t+\sigma \sqrt{\Delta t} \Phi^{-1}\left(\alpha_{1}\right)\right]-\left[v \Delta t+\sigma \sqrt{\Delta t} \Phi^{-1}\left(\alpha_{2}\right)\right]\right]=\Delta x=\ln (u)$, which simplifies to $\sigma \sqrt{\Delta t}\left[\Phi^{-1}\left(\alpha_{1}\right)-\Phi^{-1}\left(\alpha_{2}\right)\right]=\Delta x=\ln (u)$. This leads to the following expressions for the percentiles: $\alpha_{1}=\Phi\left(-\frac{v \sqrt{\Delta t}}{\sigma}-\frac{\Delta x}{\sigma \sqrt{\Delta t}}\right), \alpha_{3}=$ $\Phi\left(-\frac{v \sqrt{\Delta t}}{\sigma}+\frac{\Delta x}{\sigma \sqrt{\Delta t}}\right)$, and for the branching probabilities:

$$
\begin{aligned}
p_{1} & =\frac{1}{2}\left(\frac{\sigma^{2} \Delta t+v^{2} \Delta t^{2}}{\Delta x^{2}}-\frac{v \Delta t}{\Delta x}\right) ; p_{2}=1-\frac{\sigma^{2} \Delta t+v^{2} \Delta t^{2}}{\Delta x^{2}} ; p_{3} \\
& =\frac{1}{2}\left(\frac{\sigma^{2} \Delta t+v^{2} \Delta t^{2}}{\Delta x^{2}}+\frac{v \Delta t}{\Delta x}\right) .
\end{aligned}
$$

This set of specifications is equivalent to the Clewlow and Strickland trinomial lattice method (Clewlow and Strickland (1998), pp. 52-53) discussed in Sect. 3.1. 


\section{Case 3.2.2: Matching the first moments of the underlying asset}

If $p_{2}=0, u=e^{\lambda \sigma \sqrt{\Delta t}}$ and only the first moment of the underlying asset is matched, then the trinomial lattice degenerates to the Generalized Cox-Ross-Rubinstein binomial model. Making these substitutions into Eqs. (20) to (24) and simplifying yields the following expressions for $\alpha_{1}$ and $\alpha_{3}: \alpha_{1}=\Phi\left(-\lambda-\frac{\nu \sqrt{\Delta t}}{\sigma}\right), \alpha_{3}=\Phi\left(\lambda-\frac{\nu \sqrt{\Delta t}}{\sigma}\right)$. The branching probabilities are then implied by Eqs. (20) and (21):

$$
p_{1}=\frac{e^{(\mathrm{r}-\delta) \Delta t}-u}{d-u} ; p_{3}=\frac{e^{(\mathrm{r}-\delta) \Delta t}-d}{u-d} .
$$

This is the Generalized Cox-Ross-Rubinstein Binomial Model (Chung and Shih 2007). If $\lambda=1$, then this simplifies to the well-known CRR binomial model as a special case.

\section{Case 3.2.3: Matching the first log-transformed moments}

Our general framework can also lead to some new lattice methods. We next present such an example, which follows logic similar to the CRR model. If the same conditions as in the previous model $\left(p_{2}=0, u=e^{\sigma \sqrt{\Delta t}}\right)$ are imposed, but the first moment of the log transformed underlying asset is matched instead, then the trinomial lattice decomposes to a binomial lattice which is analogous to the CRR tree. Substituting into Eqs. (20) to (24) gives: $\alpha_{1}=\Phi\left(-1-\frac{v \sqrt{\Delta t}}{\sigma}\right), \alpha_{3}=\Phi\left(1-\frac{v \sqrt{\Delta t}}{\sigma}\right)$ and the branching probabilities are: $p_{1}=\frac{1}{2}\left[\Phi^{-1}\left(\alpha_{3}\right)\right], p_{3}=-\frac{1}{2}\left[\Phi^{-1}\left(\alpha_{1}\right)\right]$.

\section{Case 3.2.4: Matching the first two log-transformed moments}

We conclude with another example of a new approximation scheme. In this case, instead of only matching the first moment of the log transformed underlying asset as in the previous case, $p_{2}$ is chosen to be zero and the first two moments of the log transformed underlying asset are matched. This might be expected to result in a more accurate fit to the continuous distribution to be approximated. Starting with the system of matching equations and applying some algebra to Eqs. (22) and (23) leads to these expressions for the percentiles and branching probabilities: $\alpha_{1}=\Phi\left(-\frac{\ln (u)+v \Delta t}{\sigma \sqrt{\Delta t}}\right), \alpha_{3}=\Phi\left(\frac{\ln (u)-v \Delta t}{\sigma \sqrt{\Delta t}}\right), p_{1}=\frac{\Phi^{-1}\left(\alpha_{3}\right)}{\Phi^{-1}\left(\alpha_{3}\right)-\Phi^{-1}\left(\alpha_{1}\right)}$ and $p_{3}=\frac{-\Phi^{-1}\left(\alpha_{1}\right)}{\Phi^{-1}\left(\alpha_{3}\right)-\Phi^{-1}\left(\alpha_{1}\right)}$, where $\ln (u)=\sqrt{(v \Delta t)^{2}+(\sigma \sqrt{\Delta t})^{2}}$.

\section{Numerical examples of Wiener process lattice models}

To illustrate the application of our general discrete approximation based on the Wiener process, we consider the common problem of valuing financial options on underlying assets which follow GBM price processes. First, we consider a relatively simple option pricing example. This example provides an illustration of the calculations used by this approach and allows the validation of our solutions using the BSM formula. In addition, this example will be used to illustrate how this general approximation scheme facilitates the optimal construction of lattice models for specific option pricing problems and for continuous distribution fitting. 
Table 2 Correlation matrix

\begin{tabular}{llll}
\hline$\Sigma_{i, j}$ & $t_{1}=0.33$ & $t_{2}=0.67$ & $t_{3}=1.00$ \\
\hline$t_{1}=0.33$ & 1 & 0.7071 & 0.5774 \\
$t_{2}=0.67$ & 0.7071 & 1 & 0.8165 \\
$t_{3}=1.00$ & 0.5774 & 0.8165 & 1 \\
\hline
\end{tabular}

Table 3 Cholesky decomposition

\begin{tabular}{llll}
\hline$A_{i j}$ & $t_{1}=0.33$ & $t_{2}=0.67$ & $t_{3}=1.00$ \\
\hline$t_{1}=0.33$ & 1 & 0 & 0 \\
$t_{2}=0.67$ & 0.7071 & 0.7071 & 0 \\
$t_{3}=1.00$ & 0.5774 & 0.5774 & 0.5774 \\
\hline
\end{tabular}

\subsection{Calibration of Wiener process lattice models}

We begin by considering a one-year maturity at the money European call option with the current underlying asset price $S_{0}$ of $\$ 100$. To illustrate the computational approach, we assume that we will construct a trinomial tree that has three time steps, so $T=1$, $N=3$ and $\Delta t=1 / 3$. We also assume that the continuously compounded risk-free discount rate is $6 \%$ per annum, that the asset pays a continuous dividend yield of 3 $\%$ per annum, and that the volatility of the asset price, $\sigma$, is $20 \%$.

The techniques for estimating the parameters in the Black-Scholes framework have been extensively examined in the literature (e.g. Steele 2010; Hull 2006; Khaled and Samia 2010). Volatility is the only parameter required in the Black-Scholes framework which is not directly observable in the market and substantial effort has been devoted to the search for reliable volatility forecasting models (c.f. Grundy 1991; Britten-Johne and Neuberer 2000; Poon 2005; Hansen and Lunde 2006; Andersen and Bondarenko 2007). For this illustrative problem, we assume that at least one of these approaches has been applied to obtain the $20 \%$ estimate for the volatility.

\subsection{Application of the Wiener process lattice model and demonstration of convergence}

The first step in applying our approach of constructing a lattice is to calculate the correlation matrix for the Wiener Process $\left(W_{t_{1}}, \ldots, W_{t_{n}}\right)$ and the corresponding Cholesky decomposition as discussed in Sect. 3. The results are shown below in Tables 2 and 3. Notice that the non-zero row values are constants in Table 2, so the Cholesky decomposition only requires the simple calculations in column 1.

To demonstrate the construction of a symmetric Wiener process tree, we arbitrarily set $\alpha_{1}=0.05$ which implies that $\alpha_{2}=0.50, \alpha_{3}=0.95, p_{1}=p_{3}=\frac{1}{2\left(\Phi^{-1}\left(\alpha_{1}\right)\right)^{2}}=$ 0.185 , and $p_{3}=0.63$. The trinomial tree structure generates the resulting values for the Wiener process at each of $3^{3}$ endpoints. For example, the lower value for $W_{t_{1}}$ in the first node is:

$$
\sqrt{t_{1}}\left(\Phi^{-1}\left(\alpha_{1}\right)\right)=\sqrt{\frac{1}{3}}\left(\Phi^{-1}(0.05)\right)=-0.95
$$




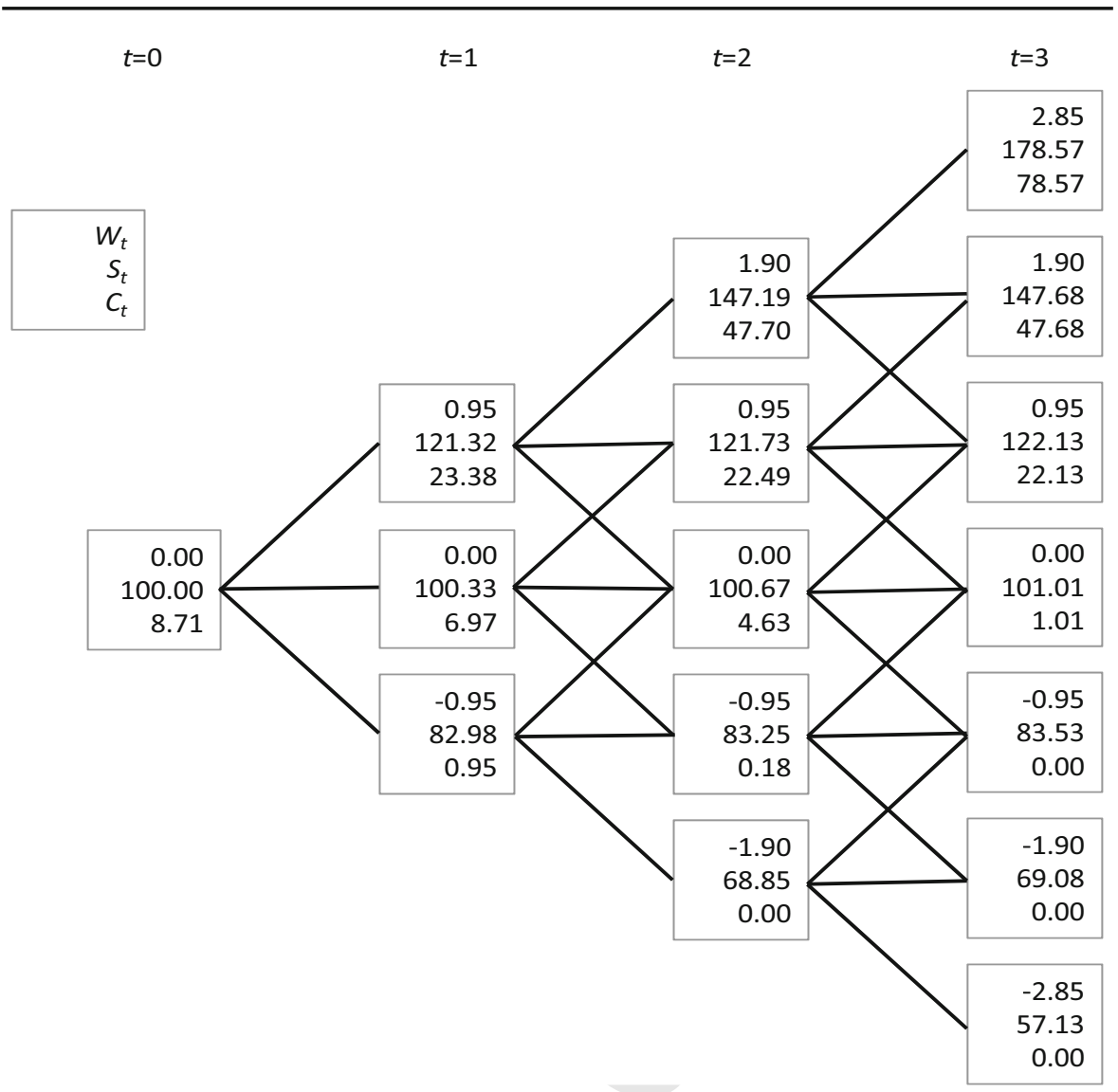

Fig. 4 Lattice for Wiener process, underlying asset price, and option price

However, this tree is symmetric and some values are recurring, so it can be reconstructed as a simple recombining lattice as shown in Fig. 4. As indicated by the key shown at the upper left of the figure, the top numbers in the outcome nodes in the lattice represent the Wiener process values, $W_{t}$.

The value of the underlying asset $S_{t}$ is then calculated as a function of the Wiener process values, using the relationship $S(t)=S(0) e^{v t+\sigma W(t)}$. Finally, the call option values at maturity are calculated and then the current option value is obtained by working backward through the structure in Fig. 4, taking discounted expectations at each node. As shown in Fig. 4 using the bottom numbers in each outcome node, the resulting European call option price is $\$ 8.71$. For comparison, the analytic solution from the BSM formula is $\$ 9.135$, where the difference is due to the relatively large time steps $(\Delta t=1 / 3$ year) which were used for simplicity in this approximation. This model converges to the BSM price as the time increments are reduced; at monthly ( $\Delta t=1 / 12$ year) increments, the difference between prices is less than five cents. 


\subsection{Optimization of the Wiener process lattice models}

There are many approaches for constructing binomial/trinomial approximations of GBM stochastic processes. It is natural to ask "Which of these approaches is the best one under certain conditions?" While the literature has shown that these lattice methods are asymptotically equivalent to the Black-Sholes model when pricing European options, and all trinomial lattices are found to be more accurate than binomial lattices (Tian 1993), there has been little discussion of the selection of a lattice approach due to the lack of a unified framework that could be used for the analysis. An important contribution of this work is that we can optimize the tree/lattice construction in a convenient manner. Furthermore, rather than being bound by the existing alternatives, we can find the exact specifications of state-space increments and branching probabilities that optimize the trinomial approximation for a specific application.

To establish the framework for the optimization of the tree/lattice construction, we first determine the decision variable. For a symmetric tree, as noted in Sect. 3.1 we can fully specify the tree/lattice structure by choosing $\alpha_{1}$, and it is thus our decision variable. There is a single constraint for this optimization, $0 \leq \alpha_{1} \leq \Phi(-1)=$ 0.1587 .

We now consider two criteria that might be used for the optimization of a trinomial lattice approximation, although we recognize that there may be other criteria that would be appropriate in specific applications. The first criterion we consider is minimization of the overall distribution fit, as measured by the root mean squared error. We then consider minimization of the option pricing and/or Greek approximation error.

\subsubsection{Selection of Wiener process lattice model by root mean square error (RMSE)}

We use root mean square error (RMSE) as a goodness-of-fit measure for the Cumulative Distribution Function (CDF) of the constructed trinomial lattice at the final stage in comparison to the theoretical underlying distribution. We define the optimal lattice as the lattice approximation with the minimum RMSE between the discrete approximation provided by the lattice and the continuous distribution available in analytical form from the stochastic process for the underlying asset in the final time period $\mathrm{T}$.

As we state more formally below, we can show that for an overall distribution fit based on optimization of RMSE, the optimal $\alpha_{1}$ and therefore the lattice structure is independent of parameters of the underlying GBM process. The intuition is based on the following observations. The lattice is constructed based upon the underlying Wiener process which is independent of parameters of the GBM process The optimal lattice structure is therefore independent of parameters of the underlying GBM process.

Theorem 2 The optimal RMSE fit for the CDF of the constructed lattice structure is independent of the parameters of the underlying GBM process.

The proof is provided in "Appendix 2".

Figure 5 shows the results of optimizing the lattice construction by minimizing the RMSE between the discrete approximation and the continuous distribution given in analytic form by the stochastic process for the underlying asset, using the example from Sect. 4.2. 


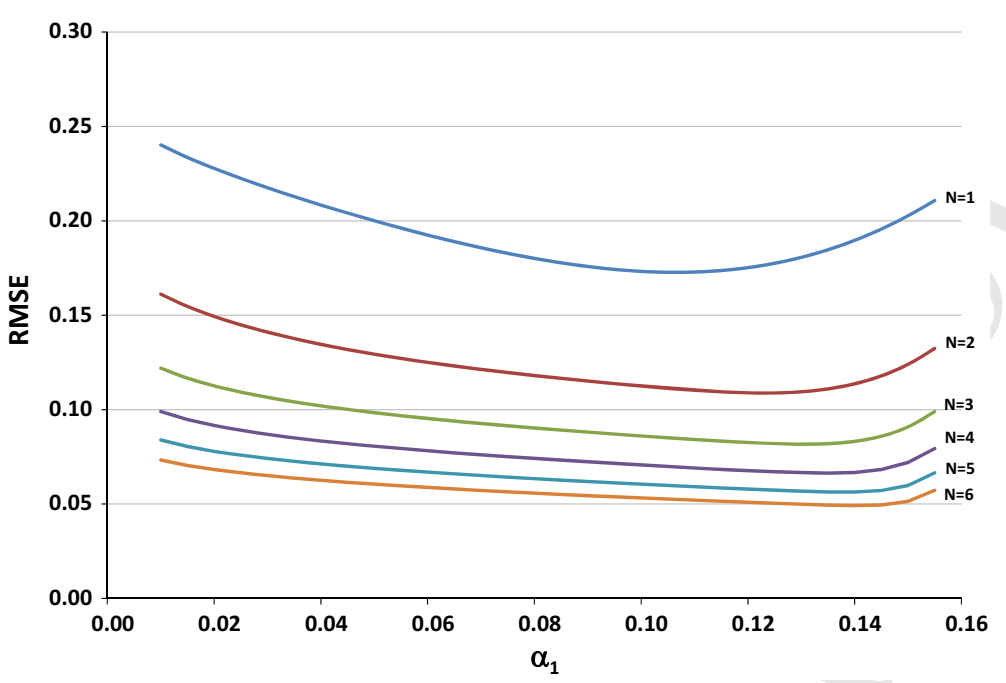

Fig. 5 Overall distribution fit optimization

As expected, as the number of time steps, $N$, increases, the accuracy of the approximation improves, as shown in Fig. 5 by the downward progression of the curves in the figure. We also observe that as $N$ increases and more endpoints are modeled along the distribution, it is less important to extend the up and down branches of the trinomial approximation in each step out into the tails of the distribution. This results in optimized $\alpha_{1}$ values progressing toward the maximum end of the range.

\subsubsection{Selection of Wiener process lattice model by Greek approximation error}

The second criterion is the minimization of the error in approximating a particular value or price which can be calculated by other means, so that a benchmark can be established for optimizing the lattice construction. For example, benchmarks for simple option prices and basic option price sensitivities ('Greeks') could be calculated using the BSM option pricing model or Monte Carlo simulation. One could then apply the optimized trinomial lattice to more complex problems than the simple ones used for benchmarking.

As we state more formally in Theorem 3, the RMSE fit for the option price and the Greeks of a simple option depends on the parameters of the underlying GBM process, as well as the choice of the parameters for the lattice approximation. This is intuitive since the benchmark option price or option price sensitivities are functions of the parameters of the underlying GBM process. Therefore the optimal lattice structure is also dependent on the parameters of the underlying GBM.

Theorem 3 The optimal RMSE approximation for option price or option price sensitivity ('Greeks') from the constructed trinomial lattice is dependent on the parameters of the underlying GBM process.

The proof is provided in "Appendix 3". 

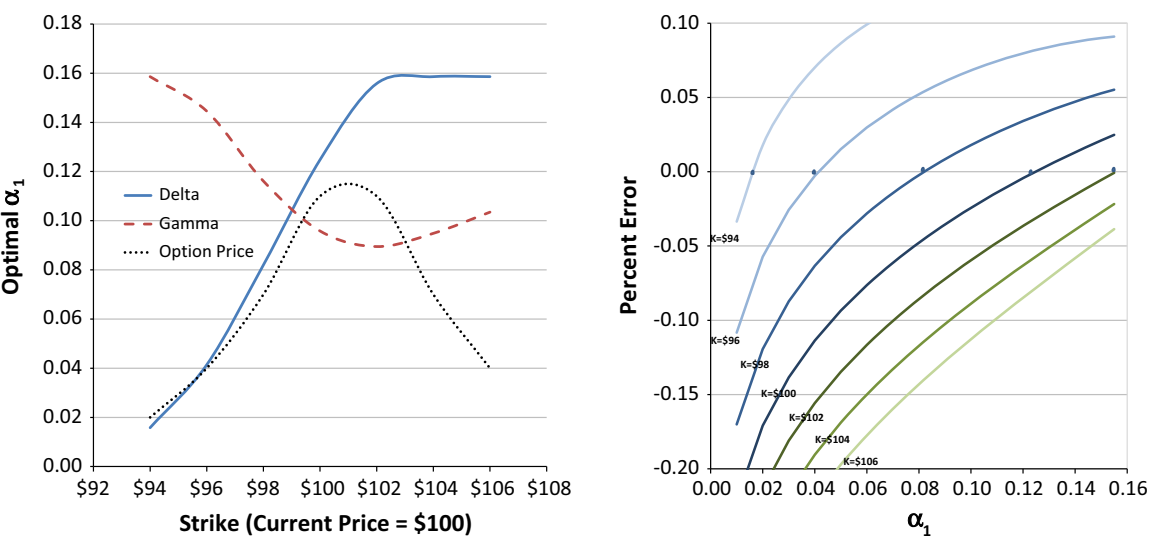

Fig. 6 Percent error in approximating option price error minimization (left), $\Delta$ (right)

Again using the example from Sect. 4.1, for a given volatility we can show numerically that the value of $\alpha_{1}$ that minimizes the error in both the option price and the Greeks varies with the relation of the current price to the strike price. The optimal value $\alpha_{1}^{*}$ versus the strike price for Delta, as well as Gamma (second-order sensitivity to underlying asset price) and the option price itself, are shown on the left panel of the Fig. 6. The shapes of the curves for $\alpha_{1}$ in this panel can be explained in the context of fitting the distribution for the underlying asset. Intuitively, the explanation for the curve showing the optimal value of $\alpha_{1}$ for approximating option price is based on the observation that option prices are best approximated when the distribution of the underlying is most accurately modeled in the tails of the distribution, especially when there is some distance between the current value and the exercise price, as demonstrated by the lower values for $\alpha_{1}^{*}$ at the right and left extremes of the dotted curve.

The solid curve showing the optimal $\alpha_{1}$ for approximating Delta illustrates Delta's dependence on the values of both the option price and the underlying asset value at the upper and lower boundaries of the discrete approximation. Specifically, as the option becomes deeper into the money, the difference between the boundary values for the option increase, while the difference between boundary values for the underlying asset do not change significantly, and the result is that Delta values increase. Because this is primarily caused by changes in the option price, the effect on $\alpha_{1}$ is similar to the effect of the option price on the in-the-money side of the plot (left side where strike price is below current price). However for out of the money cases, the differences between boundary values for the option are smaller, while the underlying asset boundary values are again similar, so the optimal values of $\alpha_{1}$ in those cases indicate that it is less important to place discrete points in the extreme regions of the distribution.

We can infer from the dashed curve showing the optimal $\alpha_{1}$ for approximating Gamma that it is most important to model values in the tails of the distribution of the underlying asset value when the value is near the strike price. This result is somewhat intuitive, since the magnitude of Gamma is highest for an at the money option.

The right panel of Fig. 6 shows the values for $\alpha_{1}$ that minimize the percent error in estimating the option price sensitivity to the underlying asset price (the Greek Delta), 

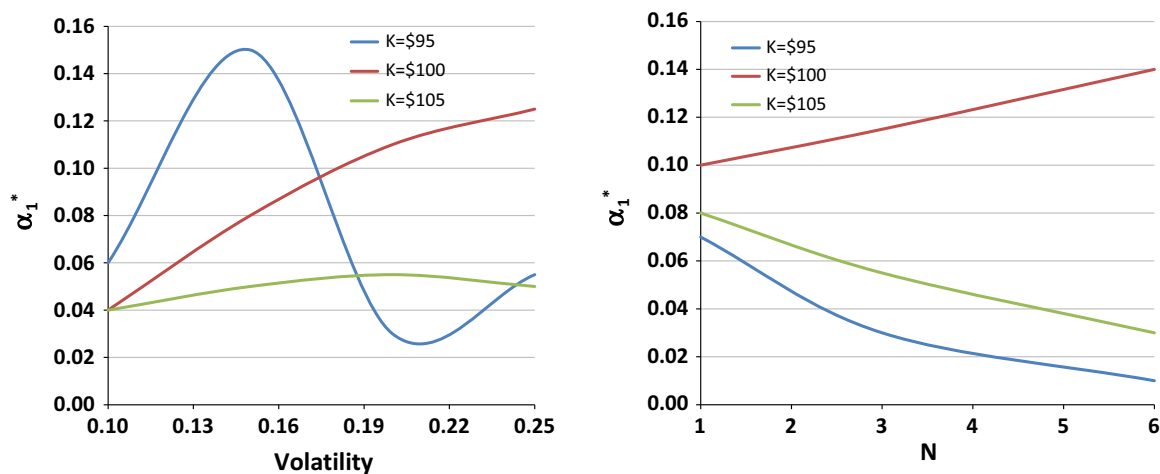

Fig. 7 Effects of volatility and number of periods on $\alpha_{1}^{*}$

as also shown in the solid blue curve in the left panel, but this panel also shows the percent error in estimating Delta with non-optimal values for $\alpha_{1}$, by tracing up or down each curve from the dot at the zero percent error point and reading the corresponding values on the left axis. These errors could be resolved by increasing the number of time periods, but this graphic illustrates how a modeler could instead choose to optimize construction of the lattice to improve accuracy.

We also might expect there to be some dependence of the optimal tree construction based on the volatility of the underlying process and the number of time increments used in the model. These two relationships, for in, at, and out of the money option cases, are shown in the left and right panels of Fig. 7, respectively.

The volatility curves in the left panel have similar starting points for low $(10 \%)$ volatility, but then vary widely and differently for the three cases. The optimal value of $\alpha_{1}$ is least sensitive to volatility for an out of the money option, where lower $\alpha_{1}$ values and more accurate modeling of values in the tails of the distribution are optimal over the entire range of volatility values. The curves for the number of periods modeled again begin from similar points for $N=1$ time periods, but for in- and out-of the money cases, as $N$ increases, it is optimal to reduce $\alpha_{1}$ and increase the number of lattice endpoints in the tails. This result is due to option value being most significantly affected in those cases by asset value realizations on the opposite sides of the distribution, such as high price realizations for an out of the money option.

\section{Conclusions}

In this paper, we have developed a general discrete approximation method based on copulas. In order to construct trinomial trees or lattices using this method, we first develop a trinomial model of the underlying Wiener process, $W(t)$. In this approach, the specifications for this model are derived by simultaneously solving for a set of six parameters for the underlying Wiener process tree. We show that this procedure provides a very general trinomial method for modeling variables $S(t) \operatorname{or} \ln (S(t))$, and that various binomial and trinomial tree methods in the literature are special cases of this approach obtained simply by the choice of the particular values of the parameters. This procedure has the potential benefit of significantly reducing computational 
costs, since it decomposes the lattice structure in such a way that all future time steps are simple multiples of values in the first time increment. This property also makes our approach very easy to implement. We have illustrated these properties and their practical implications through a set of numerical examples.

The Black-Scholes differential equation for option pricing and the previously discussed literature of parametric binomial/trinomial lattice approaches assume that the log-returns are normally distributed under the underlying GBM stochastic process. Future research in this area might include a further generalization of this framework to include other types of stochastic processes. For example, empirical studies have shown that stock returns often have fat tails and that t-distributions fit these returns better than normal distributions (e.g., Fama 1965; Praetz 1972; Zhu and Galbraith 2010). Therefore, it would be useful to extend the copula-based approach to model the stock returns as a Lévy-Student process, as discussed in Petroni et al. (2006) and Grigelionis (2013),

$$
\frac{d S(t)}{S(t)}=(r-\delta) d t+\sigma d L(t)
$$

where $d L(t)$ represents a Lévy-Student process.

To model the process for a variable $S(t)$ as a tree structure, parallel to our previous discussion on normal copulas, we could focus on the Lévy-Student process $L(t)$ as a multivariate t-distribution with underlying multivariate t-copulas. More specifically, we could utilize the multivariate t-copulas, $C_{T}\left(u_{1}, \ldots, u_{n}\right)=$ $t_{\Sigma, v}\left(t_{v}^{-1}\left(u_{1}\right), \ldots, t_{v}^{-1}\left(u_{n}\right)\right)$ where $t_{v}$ is the univariate student's $t$ distribution function, with $v$ degrees of freedom, and $t_{\Sigma, v}$ the multivariate distribution corresponding to $t_{v}$. The t-copula based dependent tree method can be used to construct the general tree structure for the Lévy-Student process following the steps discussed in details in Wang and Dyer (2012). Unlike the normal copulas case for the GBM process, however, there is no analytical solution to make this tree structure recombining by choosing values for a subset of the parameters. Future research in this area might also include further generalization of this framework to include path dependent stochastic processes such as mean reverting processes, jump diffusion processes, stochastic volatility processes, and multivariate stochastic processes.

\section{Appendix 1}

For convenience, we will prove Part $\mathrm{b}$ of Theorem 1 first:

We can show the moments of $x(t)=\ln (S(t))$ are a function of the moments of $W(t)$ by using moment generating functions. The moment generating function of the normal distribution $X \sim N\left(\mu, \sigma^{2}\right)$ is given as $M_{X}(z)=\exp \left(\mu z+\frac{1}{2} \sigma^{2} z^{2}\right)$. If $W(t) \sim N(0, t)$, then $\sigma W(t) \sim N\left(0, \sigma^{2} t\right)$, and therefore, $M_{W(t)}(z)=\exp \left(\frac{1}{2} t z^{2}\right)$, and $M_{\sigma W(t)}(z)=\exp \left(\frac{1}{2} \sigma^{2} t z^{2}\right)$

We now focus on the log-transformed asset price. For $x(t)=\ln (S(t)) \sim$ $N\left(\ln (S(0))+v t, \sigma^{2} t\right)$, we will provide the derivation of its moment generating function. 


$$
\begin{aligned}
M_{x(t)}(z) & =\mathrm{e}^{(\ln (S(0))+v t) z+\frac{1}{2} \sigma^{2} t z^{2}}=\mathrm{e}^{(\ln (S(0))+v) z} \mathrm{e}^{\frac{1}{2} \sigma^{2} t z^{2}} \\
& =\mathrm{e}^{(\ln (S(0))+v) z} \mathrm{e}^{\left(\frac{1}{2} t z^{2}\right) \sigma^{2}}=\mathrm{e}^{(\ln (S(0))+v) z}\left(M_{W(t)}(z)\right)^{\sigma^{2}} \\
& =\mathrm{e}^{(\ln (S(0))+v) z} M_{\sigma W(t)}(z)
\end{aligned}
$$

The following equation can be obtained using the Taylor expansion operation on $\mathrm{e}^{[\ln (S(0))+v t] z}$ :

$$
\mathrm{e}^{(\ln (S(0))+v) z}=1+(\ln (S(0))+v) z+\frac{((\ln (S(0))+v) z)^{2}}{2 !}+\cdots
$$

which simplifies to

$$
M_{x(t)}(z)=\left[1+(\ln (S(0))+v t) z+\frac{(\ln (S(0))+v t)^{2} z^{2}}{2 !}+\cdots\right] \times M_{\sigma W(t)}(z)
$$

If the moment generating function exists on an open interval around $t=0$, then it is the exponential generating function of the moments of the probability distribution:

$$
E\left(X^{n}\right)=M_{X}^{(n)}(0)=\frac{d^{n} M_{X}}{d z^{n}}(0)
$$

Performing the nth derivatives on the moment generating function of $x(t)$

$$
\begin{aligned}
E\left(x(t)^{n}\right)= & M_{x(t)}^{(n)}(0) \\
= & \left.\frac{d^{n}\left[1+(\ln (S(0))+v t) z+\frac{(\ln (S(0))+v t)^{2} z^{2}}{2 !}+\cdots\right]}{d z^{n}} * M_{\sigma W(t)}(z)\right|_{z=0} \\
& +\cdots \cdots+\left.\left[1+(\ln (S(0))+v t) z+\frac{(\ln (S(0))+v t)^{2} z^{2}}{2 !}+\cdots\right]\right|_{z=0} \\
& \times M_{\sigma W(t)}^{(n)}(0) \\
= & c E\left((\sigma W(t))^{n}\right) \\
= & (\ln (S(0))+v t)^{n}+[1+(\ln (S(0))+v t) z \\
& \left.+\frac{(\ln (S(0))+v t)^{2} z^{2}}{2 !}+\cdots\right]\left.\right|_{z=0} \\
& \times M_{\sigma W(t)}^{(n)}(0)=(\ln (S(0))+v t)^{n}+E\left((\sigma W(t))^{n}\right) \\
= & (\ln (S(0))+v t)^{n}+\sigma^{n} E\left((W(t))^{n}\right) \\
&
\end{aligned}
$$

Notice that the Taylor expansion only serves as an auxiliary step in the proof. The equation is exact and no approximation is required.

Proof of Part a: By the definition of a moment generating function, $M_{x(t)}(z)=$ $E\left(\mathrm{e}^{x(t) z}\right)$. Since $x(t)=\ln (S(t)), M_{x(t)}(z)=E\left(\mathrm{e}^{x(t) z}\right)=E\left(\mathrm{e}^{\ln (S(t)) z}\right)=$ 
$E\left(S(t)^{z}\right)$. Therefore, $E\left(S(t)^{n}\right)=M_{x(t)}(n)$. From the proof of Part b, we know that

$$
\begin{aligned}
E\left(S(t)^{n}\right) & =M_{x(t)}(n)=\mathrm{e}^{(\ln (S(0))+v t) n} M_{\sigma W(t)}(n)=S(0)^{n} \mathrm{e}^{v t n} \times M_{\sigma W(t)}(n) \\
& =S(0)^{n} \mathrm{e}^{v t n} \times\left(M_{W(t)}(z)\right)^{\sigma^{2}}
\end{aligned}
$$

\section{Appendix 2}

RMSE is used as a goodness-of-fit measure for the CDF of the constructed trinomial lattice at the final stage in comparison to the theoretical underlying distributions. In this case, it can be shown that for overall distribution fit based on optimization of RMSE, the optimal $\alpha_{1}$ and thence the lattice structure is independent of parameters of the underlying GBM process.

$$
\begin{aligned}
\mathrm{RMSE} & =(\mathrm{MSE})^{\frac{1}{2}} \\
\mathrm{MSE} & =\sum_{j} p_{T, j}\left(C D F^{\text {lattice }}\left(S_{T, j}\right)-C D F^{\text {theoretical }}\left(S_{T, j}\right)\right)^{2}, j=0, \ldots, 2 T
\end{aligned}
$$

Since the lattice of the GBM process is transferred from the underlying Wiener process, $C D F^{\text {lattice }}\left(S_{T, j}\right)=C D F^{\text {lattice }}\left(W_{T, j}\right)=\sum_{i}^{W_{T, i} \leq W_{T, j}} p_{T, i}$.

Therefore, either $p_{T, j}$ or $C D F^{\text {lattice }}\left(S_{T, j}\right)$ is a function of parameters of the underlying GBM process. If $C D F^{\text {theoretical }}\left(S_{T, j}\right)$ is also independent of parameters of the underlying GBM process, then MSE hence RMSE is independent of parameters of the underlying GBM process.

Since the CDF of the lognormal distribution is the same as the CDF of the normal distribution, with $\log x$ substituted for $x$,

$$
\begin{aligned}
& \mathrm{CDF}_{\text {lognormal }}(x)=\mathrm{CDF}_{\text {normal }}(\log x) \\
& C D F^{\text {theoretical }}\left(S_{T, j}\right)=C D F^{\text {theoretical }}\left(\ln \left(S_{T, j}\right)\right) \\
& \ln (S(T)) \sim N\left(\ln (S(0))+v T, \sigma^{2} T\right), \text { and } \\
& \ln \left(S_{T, j}\right)=S(0)+v T+\sigma \sqrt{T} \mathrm{~W}_{T, j} \\
& C D F^{\text {theoretical }}\left(S_{T, j}\right)=C D F^{\text {theoretical }}\left(\ln \left(S_{T, j}\right)\right) \\
& =C D F^{\text {theoretical }}\left(\frac{S(0)+v T+\sigma \sqrt{T} \mathrm{~W}_{T, j}-(S(0)+v T)}{\sigma \sqrt{T}}\right) \\
& =C D F^{\text {theoretical }}\left(\mathrm{W}_{T, j}\right) .
\end{aligned}
$$

Therefore, RMSE of the CDF of the constructed trinomial lattice at the final stage in comparison to the theoretical underlying distributions is independent of parameters of the underlying GBM process, so is the optimal $\alpha_{1}$ for overall distribution fit optimization of RMSE. 


\section{Appendix 3}

If RMSE is used as a goodness-of-fit measure for option price (e.g, call option price) from the constructed trinomial lattice at the final stage in comparison to the theoretical BSM model, the optimal lattice structure is dependent on the parameters of the underlying GBM process.

$$
\begin{aligned}
\mathrm{RMSE} & =(\mathrm{MSE})^{\frac{1}{2}} \\
\operatorname{MSE} & =\sum_{j} p_{T, j}\left(\operatorname{Max}\left(0, S_{T, j}-K\right)-B S\left(S_{0}, K, r, \delta, \sigma, T\right)\right)^{2}, j=0, \ldots, 2 T,
\end{aligned}
$$

and,

$$
S_{T, j}=e^{S(0)+\nu T+\sigma \sqrt{T} \mathrm{~W}_{T, j}}
$$

Similarly, if $\mathrm{RMSE}_{\Delta}$ is used as a goodness-of-fit measure for Greeks of option price (e.g, Delta) from the constructed trinomial lattice at the final stage in comparison to the theoretical BSM model, the optimal lattice structure is dependent on the parameters of the underlying GBM process.

$$
\begin{aligned}
\operatorname{RMSE}_{\Delta} & =\left(\mathrm{MSE}_{\Delta}\right)^{\frac{1}{2}} \\
\operatorname{MSE}_{\Delta} & =\frac{C_{T, 2 T}-C_{T, 0}}{S_{T, 2 T}-S_{T, 0}}-e^{-\delta T} N\left(d_{1}\right) \\
& =\left(\frac{\operatorname{Max}\left(0, S_{T, 2 T}-K\right)-\operatorname{Max}\left(0, S_{T, 0}-K\right)}{S_{T, 2 T}-S_{T, 0}}-\Delta_{B S}\left(S_{0}, K, r, \delta, \sigma, T\right)\right)^{2}
\end{aligned}
$$

and,

\section{Therefore, we know that RMSE (RMSE ${ }_{\Delta}$ ) will be a function of the parameters of the} underlying GBM process. The relationship between the optimal RMSE $\left(\mathrm{RMSE}_{\Delta}\right)$ and the parameters of the underlying GBM process are complex as illustrated in Sect. 4.

\section{References}

Andersen, T. G., \& Bondarenko, O. (2007). Construction and interpretation of model-free implied volatility. In I. Nelken (Ed.), Volatility as an asset class (pp. 141-181). London: Risk Books.

Barone-Adesi, G., Fusari, N., \& Theal, J. (2008). Barrier Option pricing using adjusted transition probabilities. Journal of Derivatives, 16(2), 36-53.

Baule, R., \& Wilkens, M. (2004). Lean trees-a general approach for improving performance of lattice models for option pricing. Review of Derivatives Research, 7(1), 53-72.

Britten-Johne, M., \& Neuberer, A. (2000). Option prices, implied price processes, and stochastic volatility. Journal of Finance, 55, 839-866. 
Black, F., \& Scholes, M. (1973). The pricing of options and corporate liabilities. Journal of Political Economy, 81, 637-659.

Bickel, J. Eric, Lake, L. W., \& Lehman, J. (2011). Discretization, simulation, and Swanson's (Inaccurate) mean. SPE Economics and Management, 3(3), 128-140.

Bizid, A., \& Jouini, E. (2005). Equilibrium pricing in incomplete markets. The Journal of Financial and Quantitative Analysis, 40, 833-848.

Boyle, P. P. (1988). A lattice framework for option pricing with two state variables. The Journal of Financial and Quantitative Analysis, 35, 1-12.

Broadie, M., \& Detemple, J. B. (2004). Option pricing: Valuation models and applications. Management Science, 50(9), 1145-1177.

Broadie, M., \& Kaya, Ö. (2007). A binomial lattice method for pricing corporate debt and modeling chapter 11 proceedings. Journal of Financial and Quantitative Analysis, 42(02), 279-312.

Chambers, D. R., \& Lu, Q. (2007). A tree model for pricing convertible bonds with equity, interest rate, and default risk. Journal of Derivatives, 14(4), 25-46.

Chen, R., \& Yang, T. (1999). A universal lattice. Review of Derivatives Research, 3, 115-133.

Chung, S., \& Shih, P. (2007). Generalized Cox-Ross-Rubinstein binomial model. Management Science, 53(3), 508-520.

Clewlow, L., \& Strickland, C. (1998). Implementing derivative models. New York: Wiely.

Costabile, M., Massabó, I., \& Russo, E. (2011). A binomial approximation for two-state Markovian HJM models. Review of Derivatives Research, 14(1), 37-65.

Cox, J. C., Ross, S. A., \& Rubinstein, M. (1979). Option pricing: A simplified approach. Journal of Financial Economics, 7, 229-264.

Derman, E., Kani, I., \& Chriss, N. (1996). Implied trinomial trees of the volatility smile. Quantitative Strategies Research Notes, Goldman-Sachs.

Dumas, B., \& Lyasoff, A. (2012). Incomplete-market equilibria solved recursively on an event tree. The Journal of Finance, 67, 1897-1941.

Fama, E. F. (1965). The behaviour of stock market prices. The Journal of Business, 38, 34-105.

Figlewski, S., \& Gao, B. (1999). The adaptive mesh model: A new approach to efficient option pricing. Journal of Financial Economics, 53, 313-351.

Glasserman, P. (2003). Monte Carlo methods in financial engineering. Berlin: Springer.

Grigelionis, B. (2013). Student's T-distribution and related stochastic processes. Berlin: Springer.

Grundy, B. D. (1991). Option prices and the underlying asset's return distribution. Journal of Finance, 46, 1045-1069.

Hansen, P. R., \& Lunde, A. (2006). Consistent ranking of volatility models. Journal of Econometrics, 131(1), 97-121.

He, H. (1990). Convergence from discrete- to continuous-time contingent claims prices. The Review of Financial Studies, 3, 523-546.

Hilliard, J. E., \& Schwartz, A. (2005). Pricing European and American derivatives under a jump-diffusion process: A bivariate tree approach. The Journal of Financial and Quantitative Analysis, 40(3), 671691.

Hull, J. (2006). Options futures and other derivatives (6th ed.). Englewood Cliffs, NJ: Prentice-Hall.

Hull, J. C., \& White, A. (1990). Valuing Derivative securities using the explicit finite difference method. The Journal of Financial and Quantitative Analysis, 25, 87-100.

Jabbour, G. M., Kramin, M. V., \& Young, S. D. (2010). Structural default modeling: A lattice-based approach. Journal of Derivatives, 17(4), 44-53.

Jarrow, R., \& Rudd, A. (1983). Option pricing. Homewood: R.D. Irwin.

Ji, D., \& Brorsen, B. W. (2011). A recombining lattice option pricing model that relaxes the assumption of lognormality. Review of Derivatives Research, 14(3), 349-367.

Keefer, D., \& Bodily, S. E. (1983). Three-point approximations for continuous random variables. Management Science, 29, 595-609.

Khaled, K., \& Samia, M. (2010). Estimation of the parameters of the stochastic differential equations Black-Scholes model share price of gold. Journal of Mathematics and Statistics, 6(4), 421.

Megill, R. E. (1984). An introduction to risk analysis (2nd ed.). Tulsa: PennWell.

Omberg, E. (1988). Efficient discrete time jump process models in option pricing. The Journal of Financial and Quantitative Analysis, 23, 161-174.

Parkinson, M. (1977). Option pricing: The American put. Journal of Business, 50, 21-36. 
Pearson, E. S., \& Tukey, J. W. (1965). Approximate means and standard deviations based on distances between percentage points of frequency curves. Biometrika, 52(3/4), 546-553.

Praetz, P. D. (1972). The distribution of share price changes. The Journal of Business, 45, 49-55.

Petroni, N. C., De Martino, S., De Siena, S., \& Illuminati, F. (2006). Lévy-Student processes for a Stochastic model of Beam Halos. Nuclear Instruments and Methods in Physics Research Section A: Accelerators, Spectrometers, Detectors and Associated Equipment, 561(2), 237-243.

Poon, S. (2005). A practical guide to forecasting financial market volatility. London: John Wiley.

Steele, J. M. (2010). Stochastic calculus and financial applications (Vol. 45). New York: Springer Science \& Business Media.

Tian, Y. (1993). A modified lattice approach to option pricing. Journal of Futures Markets, 13, 563-577.

Tian, Y. (1999). A flexible binomial option pricing model. Journal of Futures Markets, 19, 817-843.

Wang, T., \& Dyer, J. (2012). A Copulas-based approach to modeling dependence in decision trees. Operations Research, 60, 225-242.

Widdicks, M., Andricopoulos, A. D., Newton, D. P., \& Duck, P. W. (2002). On the enhanced convergence of lattice methods for option pricing. Journal of Futures Markets, 22(4), 315-338.

Zhu, D., \& Galbraith, J. W. (2010). A generalized asymmetric student-t distribution with application to financial econometrics. Journal of Econometrics, 157(2), 297-305. 
Journal: 11147

Article: 9111

\section{Author Query Form}

\section{Please ensure you fill out your response to the queries raised below and return this form along with your corrections}

\section{Dear Author}

During the process of typesetting your article, the following queries have arisen. Please check your typeset proof carefully against the queries listed below and mark the necessary changes either directly on the proof/online grid or in the 'Author's response' area provided below

\begin{tabular}{|c|l|l|}
\hline Query & \multicolumn{1}{|c|}{ Details required } & \multicolumn{1}{c|}{ Author's response } \\
\hline 1. & $\begin{array}{l}\text { Please provide JEL codes. } \\
\text { For more details, please visit } \\
\text { http://www.aeaweb.org/journal/jel_cla\$s_system.html. }\end{array}$ & G13, C10 \\
\hline 2. & $\begin{array}{l}\text { Glasserman (2000) has been changed } \\
\text { as Glasserman (2003). Please check } \\
\text { and confirm. }\end{array}$ & Confirmed. Glasserman (2003) is correct. \\
\hline 3. & $\begin{array}{l}\text { Reference Hull and White (1990) is } \\
\text { given in list but not cited in text. Please } \\
\text { check and provide citation in text. }\end{array}$ & $\begin{array}{l}\text { Please delete Hull and White (1990) } \\
\text { from the list }\end{array}$ \\
\hline
\end{tabular}

